\title{
BMJ Open Effect of frailty on quality of life in elderly patients after hip fracture: a longitudinal study
}

Cornelis L P van de Ree, ${ }^{\oplus}$ Maud J F Landers, ${ }^{1}$ Nena Kruithof, ${ }^{1}$ Leonie de Munter, ${ }^{1}$ Joris P J Slaets, ${ }^{2,3}$ Taco Gosens, ${ }^{1,4}$ Mariska A C de Jongh ${ }^{1,5}$

To cite: van de Ree CLP, Landers MJF, Kruithof N, et al. Effect of frailty on quality of life in elderly patients after hip fracture: a longitudinal study. BMJ Open 2019;9:e025941. doi:10.1136/ bmjopen-2018-025941

- Prepublication history and additional material are published online only. To view please visit the journal online (http://dx.doi. org/10.1136/bmjopen-2018025941).

Received 28 August 2018 Revised 20 February 2019 Accepted 14 June 2019

Check for updates

(C) Author(s) (or their employer(s)) 2019. Re-use permitted under CC BY-NC. No commercial re-use. See rights and permissions. Published by BMJ.

${ }^{1}$ Trauma TopCare, ElisabethTweeSteden Ziekenhuis, Tilburg, The Netherlands

${ }^{2}$ Leyden Academy on Vitality and Ageing, Leiden, The Netherlands ${ }^{3}$ Department of Internal Medicine, Universitair Medisch Centrum Groningen, Groningen, The Netherlands

${ }^{4}$ Department of Orthopaedic Surgery, Elisabeth-TweeSteden Ziekenhuis, Tilburg, The Netherlands

${ }^{5}$ Brabant Trauma Registry, Network Emergency Care Brabant, Tilburg, The Netherlands

Correspondence to Drs. Cornelis L P van de Ree; M.vanderee@etz.nl

\section{ABSTRACT}

Objectives The aims of this study were to examine the pattern of changes over time in health status (HS) and quality of life (QoL) in the first year after hip fracture and to quantify the association between frailty at the onset of hip fracture and the change in HS and QoL 1 year later. The major hypothesis was that frailty, a clinical state of increased vulnerability, is a good predictor of QoL in patients recovering from hip fracture.

Design Prospective, observational, follow-up cohort study. Setting Secondary care. Ten participating centres in Brabant, the Netherlands.

Participants 1091 patients entered the study and 696 patients completed the study. Patients with a hip fracture aged 65 years and older or proxy respondents for patients with cognitive impairment were included in this study.

Main outcome measures The primary outcomes were HS (EuroQol-5 Dimensions questionnaire) and capability well-being (ICEpop CAPability measure for Older people). Prefracture frailty was defined with the Groningen Frailty Indicator (GFI), with GFI $\geq 4$ indicating frailty. Participants were followed up at 1 month, 3 months, 6 months and 1 year after hospital admission.

Results In total, 371 patients (53.3\%) were considered frail. Frailty was negatively associated with HS ( $\beta-0.333$; $95 \% \mathrm{Cl}-0.366$ to -0.299$)$, self-rated health $(\beta-21.9$; $95 \% \mathrm{Cl}-24.2$ to -19.6$)$ and capability well-being ( $\beta$ $-0.296 ; 95 \% \mathrm{Cl}-0.322$ to -0.270 ) in elderly patients 1 year after hip fracture. After adjusting for confounders, including death, prefracture HS, age, prefracture residential status, prefracture mobility, American Society of Anesthesiologists grading and dementia, associations were weakened but remained significant.

Conclusions We revealed that frailty is negatively associated with QoL 1 year after hip fracture, even after adjusting for confounders. This finding suggests that early identification of prefracture frailty in patients with a hip fracture is important for prognostic counselling, care planning and the tailoring of treatment.

Trial registration number NCT02508675

\section{INTRODUCTION}

A hip fracture is a serious event in the elderly population. It is associated with high mortality, morbidity and disability for those who survive. ${ }^{1-3}$ Hip fracture risks rise exponentially with increasing age. With the rising

\section{Strengths and limitations of this study}

- This study addresses the paucity of knowledge on frailty in elderly patients with a hip fracture.

- This multicentre, prospective cohort study included a large number of subjects.

- Patients and proxy participants from different geographical locations were included, which increases the generalisability of this study.

- Participants may not accurately recall their health status prior to the fracture, which might affect the results.

- The frail group contained more no-show cases, which could have resulted in selective dropout.

longevity across the globe, it seems reasonable that hip fractures will remain an important global health problem with substantial socioeconomic costs. ${ }^{45} \mathrm{~A}$ hip fracture has a major impact on health status (HS) and quality of life (QoL). ${ }^{6}$ HS represents the perceived impact of a disease on the level of patients' physical, emotional and social functioning. ${ }^{7}$ Several factors are negatively associated with HS in elderly patients with a hip fracture, including female gender, comorbidity, poor nutritional status, severe postsurgical pain perception, long duration of hospital stay, postoperative complications, and low physical or psychosocial functioning at prefracture, including cognitive dysfunction. ${ }^{6}$ QoL is a multidimensional concept including both positive and negative aspects of life, and it measures patients' evaluation of functioning in line with their expectations. ${ }^{8}$ QoL in older people is limited by an individual's loss of ability to pursue different attributes with regard to attachment, role, enjoyment, security and control. ${ }^{9}$ This multidimensional concept can be measured with a capability well-being instrument in frail older adults following a hip fracture. ${ }^{1011}$

Inconclusive evidence was found for the predictive value of older age. ${ }^{6}$ However, 
ageing is associated with a decline in physiological reserves, which impedes the body's ability to withstand and recover from major and minor challenges, for example, a hip fracture. This phenomenon is defined as frailty, a clinical state of increased vulnerability, and it interacts with psychological factors, such as emotional state, coping style and sociological state. ${ }^{12}$

A systematic review from Lin and colleagues ${ }^{13}$ demonstrated that frailty is associated with adverse outcomes in older postsurgery patients, including prolonged length of stay, complications and postoperative mortality. ${ }^{1}$ However, the relationship between frailty and HS, and between frailty and capability well-being, is unknown. The aims of this study were to (1) compare HS by frailty status at the time of hip fracture, (2) describe the patterns of HS and capability well-being in the first year after hip fracture, and (3) quantify the association between frailty at the onset of hip fracture and the patterns in HS and capability well-being 1 year following a hip fracture. We hypothesised that frail hip-fractured patients would experience a higher likelihood of poor HS and capability wellbeing, even after accounting for traditionally measured clinical risk factors.

\section{MATERIALS AND METHODS}

\section{Study design and participants}

The Brabant Injury Outcome Surveillance (BIOS), a multicentre, prospective, observational, follow-up cohort study, was conducted to obtain data at 1 week and at 1 , 3, 6 and 12 months after hip fracture. Full details of the study, objectives and methods are described in detail elsewhere. ${ }^{14}$ This report has been prepared in accordance with the Strengthening the Reporting of Observational Studies in Epidemiology guidelines. ${ }^{15}$

All participants were included between August 2015 and November 2016 from the 10 participating Dutch hospitals and were invited during hospital admission or within several days post-trauma by mail. Both patients aged 65 years and older and proxy respondents for patients with cognitive impairment were eligible for inclusion. Proxy participants could participate from 1 month onwards. Exclusion criteria were as follows: (1) pathological hip fractures, (2) patients and proxy respondents being unable or unwilling to give written informed consent, and (3) patients with insufficient knowledge of the Dutch language.

\section{Data collection}

Baseline prefracture information (T0) was gathered 1 week or 1 month after hip fracture by self-reported or proxy-reported questionnaires. The following data were collected at baseline within 1 month after hip fracture: demographic characteristics (age, gender, educational level), American Society of Anesthesiologists (ASA) grading, mobility, degree of frailty and HS. All participants were followed up at 1 week (T1), 1 month (T2), 3 months (T3), 6 months (T4) and 1year (T5) after hospital admission. At follow-up sessions, questionnaires were sent to the participant or proxy. In cases of no return, they were contacted by telephone several times. If this method failed, the participant or proxy was considered to be a non-responder at that follow-up time point.

\section{Patient and public involvement}

Patients were involved in the recruitment to and conduct of the study. In a small pilot before inclusion in the BIOS, patients were asked their findings about the questionnaire and outcomes. We made small adjustments and results were disseminated to study participants who want to receive information by a newsletter.

\section{Outcome assessment questionnaires}

The Groningen Frailty Indicator (GFI) questionnaire was used to identify elderly individuals as being frail. The GFI is a 15-item self-reported instrument and screens for the loss of function and resources in four domains of functioning: physical, cognitive, social and psychological (online supplementary file). ${ }^{16}$ The sum score of the GFI ranges from 0 to 15 , with a score of $\geq 4$ indicating frailty. The study of Peters $e t a l^{17}$ concluded that the GFI is a feasible, reliable and valid self-assessment in homedwelling and institutionalised elderly people by detecting those at high risk for poor outcomes. ${ }^{1}$

The score on the EuroQol-5 Dimensions (EQ-5D) is a measure of HS. ${ }^{18}$ The EQ-5D has two parts: Visual Analogue Scale (VAS), which measures self-rated health, and an instrument along five health domains related to daily activities, including mobility, self-care, usual activities, pain and discomfort, and anxiety and depression. A respondent's EQ-VAS presents self-rated health on a vertical scale with two endpoints, that is, 'best imaginable health state' (100) and 'worst imaginable health state' (0). Each dimension consists of a three-level response: no problems, moderate problems or severe problems. A scoring algorithm is available by which each HS description can be expressed into an overall score using a published utility algorithm for the Dutch population. HS was assessed with the utility score (EQ-5D utility), ranging from 0 representing death to 1 for full health. A negative utility score indicates an HS worse than death. The Dutch tariffs were used for this study to calculate EQ-5D-3 Level preference weights. ${ }^{19}$ The EQ-5D has good measurement properties and could be used to measure outcomes for patients recovering from hip fracture. ${ }^{11}$

The ICEpop CAPability measure for Older people (ICECAP-O) provides a broad assessment of capability well-being as it measures an individual's ability to 'do' and 'be' the things that are important in life. ${ }^{20}$ This index of capability focuses on well-being defined in a broader sense, rather than defined by health, and covers the following five attributes: attachment (love and friendship), security (thinking about the future without concern), role (doing things that make you feel valued), enjoyment (enjoyment and pleasure) and control (independence). These attributes are used to calculate a tariff 
between 0 , meaning no capability, and 1 , representing full capability. The ICECAP-O has been validated in different elderly populations, and for this study the population of Makai et al of posthospitalised older people in the Netherlands was used to compare scores..$^{2122}$ The questionnaire shows good convergent validity with health and well-being instruments and is able to discriminate between elderly individuals with various health profiles. ${ }^{21} 2324$

\section{Statistical analysis}

The descriptive statistics of the cohort were presented as means with SDs for continuous variables and as numbers and percentages for dichotomous or categorical variables. Missing baseline characteristics and missing sum scores in EQ-5D and ICECAP-O were imputed according to multiple imputation, using the multivariate imputation by chained equations procedure..$^{25}$ There were no variables with $5 \%$ or more missing values. The data set was imputed 15 times with 5 iterations. Patient demographics (age, gender) were compared between responders and non-responders. Univariate and multivariable linear regression models were used to compare HS by frailty status at time of hip fracture. To assess the association between frailty and QoL over 1 year, we used linear mixed model analyses for EQ-5D utility scores and ICECAP-O scores, and we used binary logistic mixed model analyses for domains of the EQ-5D. Multicollinearity was assessed with the variance inflation factor (VIF). After univariate analyses, we performed adjusted analyses in which confounders (prefracture HS, sociodemographic variables and comorbidity) were included in the model. Because the mortality of study participants caused dropout (loss to follow-up), we performed death-adjusted analyses to adjust for overly optimistic estimates of patient outcomes. According to Parsons $e t a l^{26}$, we assumed that the EQ-5D score ranges from zero to death; these observations were then carried forward to subsequent assessment occasions. ${ }^{26}$ Effects were expressed as regression coefficients (beta; $\beta$ ), ORs and adjusted ORs (aORs) with 95\% CIs, representing the longitudinal association between frailty and HS and between frailty and capability well-being over time, reflecting both the within-subject and between-subject relationship. ${ }^{27}$ Statistical test results were considered significant at a level of $\mathrm{p}<0.05$. Statistical analyses were performed in SPSS V.24.0 and R V.3.4.0 (The R Project for Statistical Computing).

\section{RESULTS}

\section{Study population}

Figure 1 shows the flow diagram of study participants. Only patients who completed the prefracture questionnaire, including the GFI, were included in this study. No significant differences were found in patient demographics (age: $p=0.215$; sex: $p=0.183$ ) between responders and non-responders. In total, 696 patients were included, and 371 patients $(53.3 \%)$ were considered frail. Table 1 shows patients' characteristics and clinical parameters, divided into frail and non-frail participants. In total, the mean age was 80.3 years, and $70.4 \%$ of the sample were female. Furthermore, $216(31.0 \%)$ proxy participants were included.

\section{The longitudinal association between frailty and HS}

There were significant differences in HS between frail and non-frail patients during all follow-up time points $(p<0.0001$; figure 2). Prefracture frailty was associated with prefracture HS, adjusted for residential status as a confounder $(\beta-0.29$; SE $0.02 ; \mathrm{p}<0.001 ; 95 \%$ CI -0.33 to -0.26 ).

The pattern of recovery trajectories in the prevalence of reported problems in the domains of the EQ-5D during the first year period after hip fracture differed between the frail and non-frail patients (figure 3A,B). For prefracture, a significantly higher proportion of patients in the frail group had problems with mobility, self-care and usual activities, and experienced more pain and signs of anxiety/ depression $(\mathrm{p}<0.001$; table 2$)$. The percentage of patients with problems of anxiety/depression in the frail group was $54.7 \%$ at 1 week and $58.3 \%$ at 1 year, compared with $18.9 \%$ at 1 week and $14.2 \%$ at 1 year in the non-frail group. The aOR of the anxiety/depression domain revealed a 1.346fold increase in problems (95\% CI 1.045 to 1.734) experienced by frail patients over 1year, compared with the problems in the non-frail group.

The VIF before the final model analysis ranged from 1.23 to 1.69 , indicating that there was no problem with multicollinearity. Frailty was negatively associated with HS ( $\beta-0.333 ; 95 \%$ CI -0.366 to -0.299$)$ and self-rated health $(\beta-21.9 ; 95 \%$ CI -24.2 to -19.6$)$ in elderly patients 1 year after hip fracture (table 3 ). The estimated crude regression coefficient of -0.333 for frail patients in relation to HS can be interpreted as follows: a patient considered to be frail at baseline has a 0.333 lower EQ-5D utility score compared with non-frail patients. The regression coefficient was -0.115 (95\% CI -0.160 to -0.069$)$ for the association between frailty and HS, adjusted for deceased dropouts and for confounders, including prefracture EQ-5D score, age, prefracture residential status, prefracture mobility, ASA grading and dementia.

\section{The longitudinal association between frailty and capability well-being}

Figure 4 shows differences in capability well-being between frail and non-frail patients during all follow-up time points $(p<0.0001)$. We found a significantly strong negative association on average between frailty and capability well-being over time, with a death-adjusted regression coefficient that included all confounders of $\beta-0.146$ (95\% CI -0.187 to -0.106 ; table 3 ).

\section{DISCUSSION}

\section{Summary of results}

It is well known that elderly patients with a hip fracture have poor QoL. ${ }^{6}$ However, it is unknown how much frailty affects patients' QoL. This longitudinal cohort study shows that (1) frail patients with a hip fracture had 


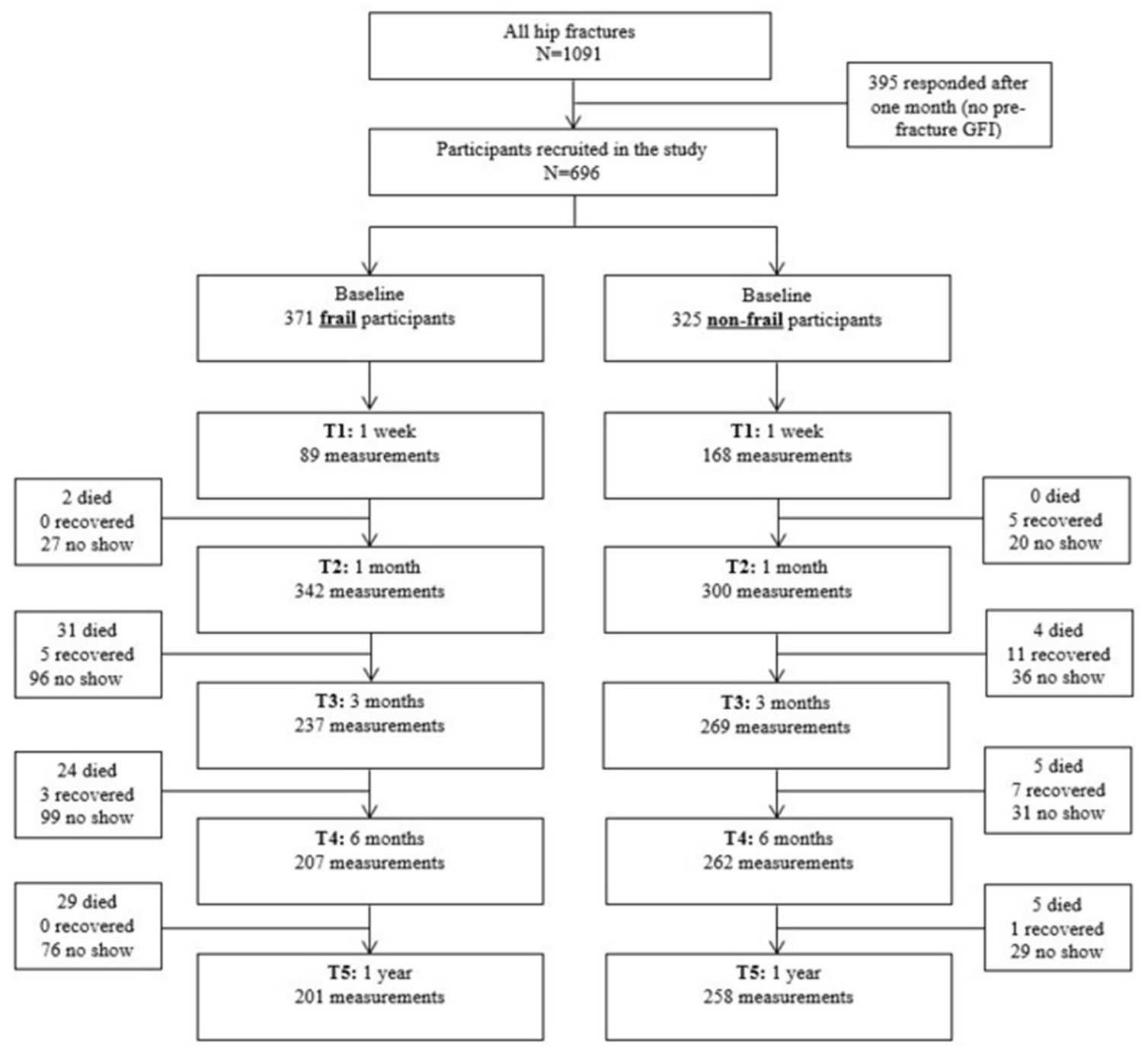

Figure 1 Flow diagram of study participants. Participants who missed some of the measurements are indicated as 'no show'. GFI, Groningen Frailty Indicator; T1, 1 week; T2, 1 month; T3, 3 months; T4, 6 months; T5, 1 year.

poorer HS than non-frail patients at baseline, (2) frail patients had poorer HS and poorer capability well-being than non-frail patients over time, and (3) frailty at the onset of hip fracture was negatively associated with HS and capability well-being 1 year after hip fracture. The pattern of recovery trajectories in the prevalence of reported problems in the domains of the EQ-5D during the first year period after hip fracture differed between the frail and non-frail patients. However, after adjustment for confounders, especially for the concerned prefracture status of the EQ-5D domain, the major differences between frail and non-frail patients disappeared. Confounders, such as prefracture HS, age, prefracture residential status, prefracture mobility, ASA grading and dementia, also weakened the association between frailty and QoL, but the association remained significant and clinically relevant. Our findings demonstrate that prefracture frailty is significantly associated with poor HS, self-rated health and capability well-being the first year after recovery from hip fracture.

\section{Comparison with existing literature}

This study demonstrates that frailty is a common condition among elderly patients with a hip fracture. In our study, 53.3\% of the patients with a hip fracture were considered frail. This finding is in line with that of a small pilot study of Kistler $e t a l,{ }^{28}$ who found that $51 \%$ of patients were considered frail. ${ }^{28}$ Previous studies, summarised in a systematic review by Lin and colleagues, showed frailty to be associated with adverse outcomes, such as prolonged length of stay and mortality in older surgical patients. ${ }^{13}$ This finding is in line with ours, showing a significant difference in length of stay between frail and non-frail patients $(\mathrm{t}(696)=-5.845, \mathrm{p}<0.001)$. In line with the findings of Patel et $a l^{29}$ and Dayama $e t a l^{30}$ we also found increased 1-year mortality rates in frail patients with a hip fracture. However, apart from these associations, our results showed that frailty is also negatively associated with QoL. This finding is of major importance because frailty seems to influence patients' postoperative outcomes, such as mortality and complications, and has a perceived 
Table 1 Demographic and clinical baseline characteristics of the cohort

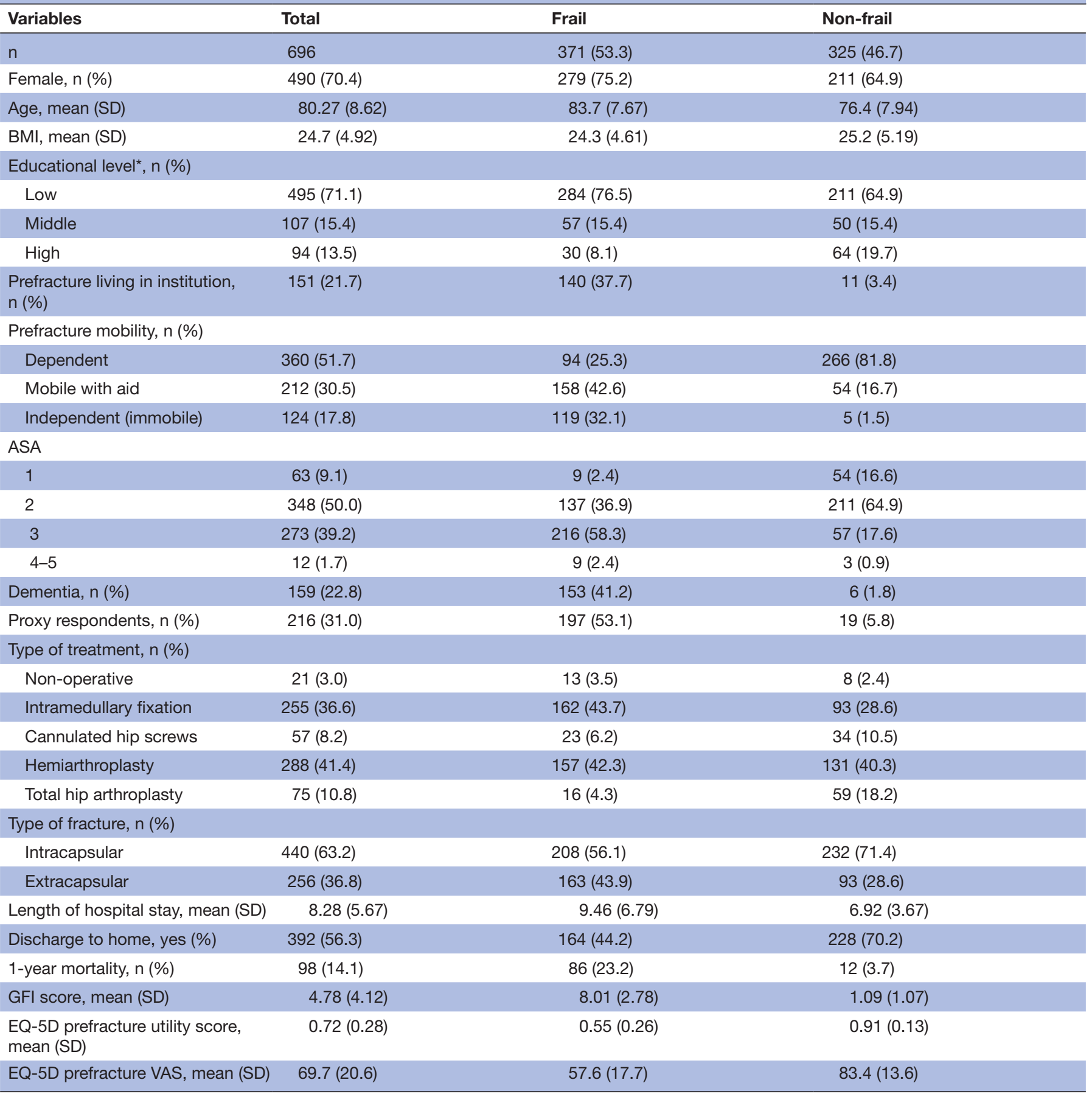

${ }^{\star}$ Educational level: low: no diploma, primary education, preparatory secondary vocational education; middle: university preparatory education, senior general secondary education, senior secondary vocational education and training; high: universities of applied sciences: associate degree or university degree.

ASA, American Society of Anesthesiologists grading; BMI, body mass index; EQ-5D, EuroQol-5 Dimensions; GFI, Groningen Frailty Indicator; VAS, Visual Analogue Scale.

impact on the level of patients' physical, emotional and social functioning. In the Netherlands, there is no difference in postfracture treatments between frail and non-frail patients. However, frail patients have already prefracture more problems with their mobility and selfcare, and therefore this could have influenced their postfracture rehabilitation possibilities.
In our study, HS and capability well-being do not generally fully recover within 12 months after hip fracture for both frail and non-frail patients. This finding is in line with that of the prospective cohort study of Griffin et al, ${ }^{31}$ who also revealed an initial marked decline in HS after hip fracture, followed by improvement within 4 months and no return to baseline at 1 year after hip fracture. ${ }^{31}$ 


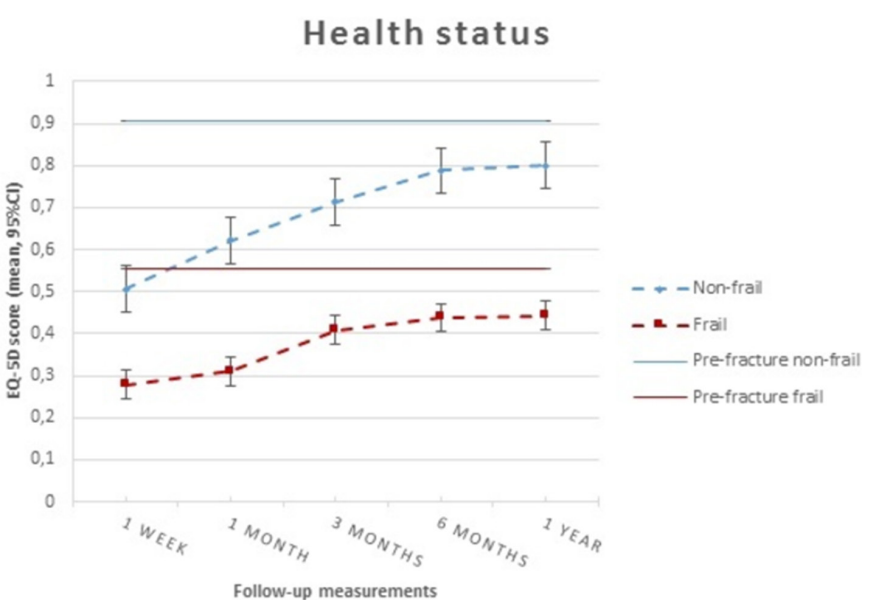

Figure 2 Patterns of health status according to frailty status over time. EQ-5D, EuroQol-5 Dimensions.

This is also in line with the International Costs and Utilities Related to Osteoporotic Fractures Study. ${ }^{32} 33$ However, in our study, we showed the pattern of QoL and distinguished between frail and non-frail patients. We revealed a significantly more prominent decline in HS, self-rated health and capability well-being for frail patients compared with non-frail patients the first year of recovery from hip fracture. To show that our findings are clinically relevant, Walters and Brazier ${ }^{34}$ published the
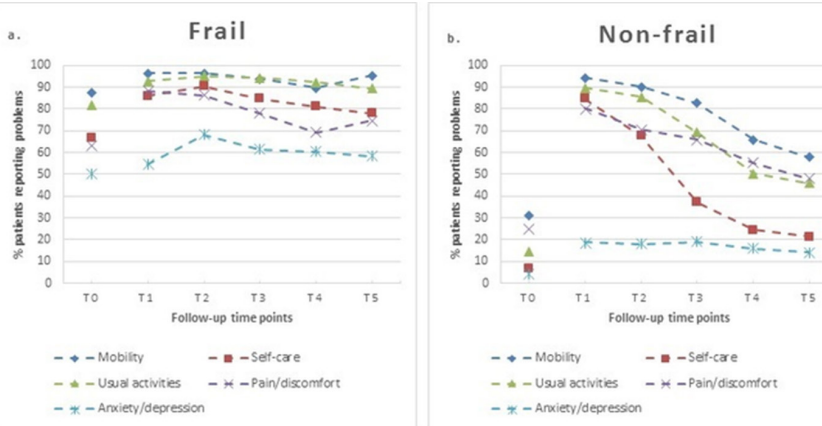

Figure 3 Percentage of frail $(A)$ and non-frail $(B)$ patients reporting problems on each EuroQol-5 Dimensions 3 Level. Questionnaire item at each follow-up time point. minimum clinically important difference of 0.074 for the utility score of the EQ-5D. ${ }^{34}$

It is remarkable that in the non-frail group, a high percentage of individuals do not return to prefracture levels within a year on all domains of the EQ-5D. In particular, the domains mobility, pain and usual activities showed major differences between the percentage of non-frail patients and that of frail patients reporting problems at baseline and 1 year after hip fracture. However, the same did not apply to the EQ-5D domain anxiety and depression, which revealed a strong positive association between frailty and anxiety/depression. Until now, the literature revealed a prevalence rate of $10 \%$ of patients reporting depressive symptoms after hip fracture. ${ }^{35}$ Future research should provide insight into whether frailty is a predictor of psychological distress, characterised by symptoms of anxiety, symptoms of depression and symptoms of post-traumatic stress.

\section{Limitations and strengths}

This study had several limitations. First, participants may not accurately recall their status prior to the fracture, which might affect the results of the GFI and the EQ-5D at baseline. To minimise recall bias, the prefracture frailty status and HS data were only collected in patients included in the study until 1 month had passed. In addition, because of the length of the questionnaire, we did not ask the items of the ICECAP-O prior to the fracture, and we could not compare this longitudinal outcome with prefracture capability well-being. Second, frail patients showed a higher capability well-being score at 1-week follow-up than at 1-month follow-up. This is probably due to selection bias because frail patients in relatively good condition were able to complete the questionnaire at this early follow-up time point. Furthermore, there were more no-show cases in the frail group, which could have resulted in selective dropout. Therefore, the overall QoL of patients after a hip fracture, especially in the frail group, is probably worse than that presented in this study. On the other hand, an early follow-up time point at 1 week is unique in prospective research in hip fracture populations, and we adjusted for confounding variables

Table 2 Mixed model analyses of change in EuroQol-5 Dimensions-3 Level for frail patients compared with non-frail patients over time

\begin{tabular}{|c|c|c|c|c|c|c|}
\hline \multirow[b]{2}{*}{ EQ-5D domain } & \multicolumn{2}{|l|}{ Crude } & \multirow[b]{2}{*}{$P$ value } & \multicolumn{3}{|c|}{ Adjusted* } \\
\hline & OR & $95 \% \mathrm{Cl}$ & & OR & $95 \% \mathrm{Cl}$ & P value \\
\hline Mobility & 1.970 & 1.501 to 2.590 & $<0.001$ & 1.186 & 0.877 to 1.605 & 0.268 \\
\hline Usual activities & 2.545 & 1.909 to 3.393 & $<0.001$ & 1.165 & 0.859 to 1.579 & 0.326 \\
\hline Pain/discomfort & 1.394 & 1.089 to 1.785 & 0.008 & 1.179 & 0.909 to 1.529 & 0.214 \\
\hline
\end{tabular}

Reference group: non-frail.

*Adjusted for prefracture status of the EQ-5D domain, age, prefracture residential status, ASA grading and dementia.

ASA, American Society of Anesthesiologists; EQ-5D, EuroQol-5 Dimensions. 
Table 3 Analyses results on the association between frailty and health status/capability well-being over 1 year after hip fracture

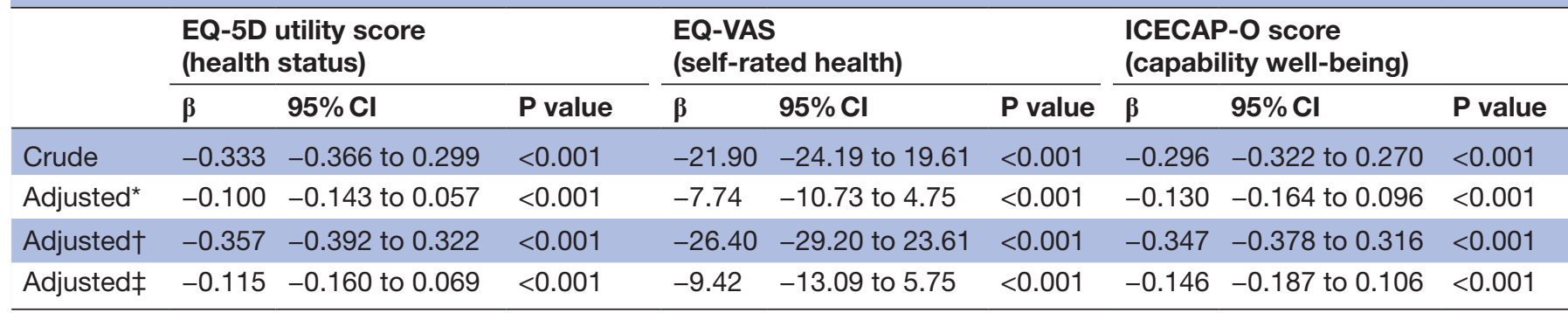

Reference group: non-frail.

*Adjusted for prefracture EQ-5D utility score, age, prefracture residential status, prefracture mobility, ASA grading and dementia. †Adjusted for death.

$\ddagger$ Adjusted for death and prefracture EQ-5D utility score, age, prefracture residential status, prefracture mobility, ASA grading and dementia. ASA, American Society of Anesthesiologists; $\beta$, regression coefficient; EQ-5D, EuroQol-5 Dimensions; EQ-VAS, EuroQol Visual Analogue Scale; ICECAP-O, ICEpop CAPability measure for Older people.

in our mixed model analyses. Third, it is well known that surgery for hip fractures is frequently followed by complications. ${ }^{36}$ However, information about complications after hip fractures was not collected in this multicentre study, and complications could have affected patients' QoL.

A strength of this study is the set-up in the form of a multicentre, prospective cohort study. We could include a large number of participants from different geographical locations, along with the possibility of including a wider range of hip fracture population groups, which increases the generalisability of this study. We also included proxy participants in case a patient was unable to participate in this study for several reasons, including cognitive impairment. Particularly, it is essential to include this group in this study because a major proportion of the frail group $(41.2 \%)$ was suffering from dementia. Gabbe et $a l^{87}$ published a study on trauma patients showing that differences in HS between patient and proxy respondents showed random variability rather than systematic bias. They concluded that group comparisons using proxy

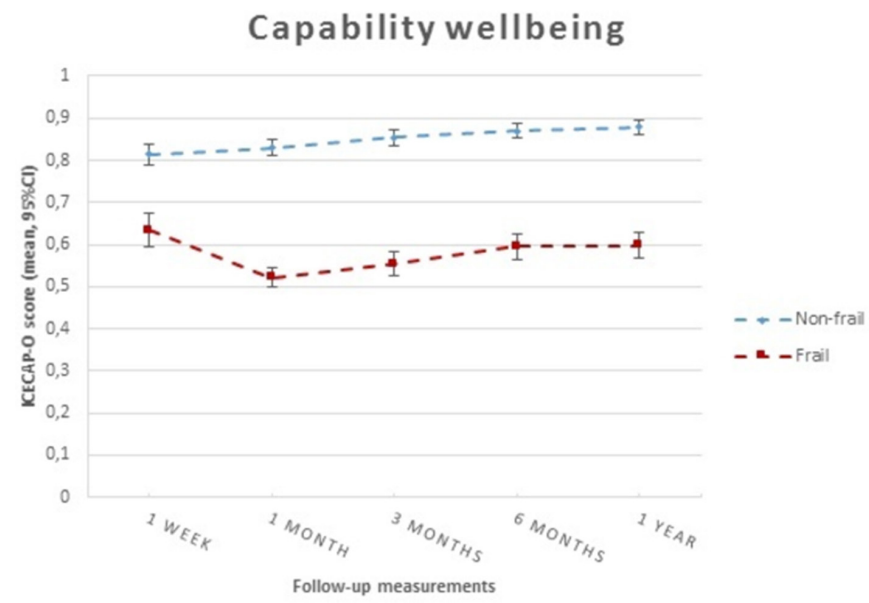

Figure 4 Patterns of capability well-being according to frailty status over time. ICECAP-O, ICEpop CAPability measure for Older people. responses are unlikely to be biased. Another strength of this study is that we reported death-adjusted outcomes according to Parsons et al. ${ }^{26}$ When reporting QoL for patients after a hip fracture, excluding patients who die during follow-up leads to overly optimistic estimates of patient outcomes and is likely to cause bias.

\section{Implication for clinical practice}

The findings of this study support the hypothesis that prefracture frailty has an unfavourable effect on HS, selfrated health and capability well-being after a hip fracture. Preoperative frailty assessment can be valuable in informing patients and their relatives about the impact of hip fracture on patients' physical, emotional and social functioning in the recovery period after a hip fracture. This frailty assessment could classify patients at high risk for unfavourable outcomes regarding poor QoL. It could support clinicians in tailoring treatment for medical decision making at an early phase. A clinically easy-to-use and universal frailty indicator, such as the GFI, could have important implications in prognostic counselling and care planning among older adults with hip fracture.

\section{CONCLUSIONS}

Our results show that frailty is negatively associated with patients' QoL 1 year after hip fracture, even after adjusting for prefracture HS, age, prefracture residential status, prefracture mobility, ASA grading and dementia. This study highlights hip fracture as a major cause of burden and morbidity, especially in frail patients. This finding suggests that early identification of prefracture frailty in patients with a hip fracture is important for prognostic counselling, care planning and the tailoring of treatment.

Contributors CvdR, NK, LdM, TG and MACJ contributed to conception and design of this study. CvdR, MJFL, NK and LdM contributed to data collection. CvdR, MJFL, NK, LdM and MACJ contributed to analyses and interpretation. CvdR, MJFL, NK, LdM, JPJS, TG and MACdJ contributed to preparation of the manuscript. The final version of the article was approved by all the authors. 
Funding This work was funded by a grant from the Netherlands Organisation for Health Research and Development (ZonMW; 842004005) TopCare projects section. This funding source did not play a role in the investigation.

Competing interests None declared.

Patient consent for publication Not required.

Ethics approval All procedures performed in studies involving human participants were in accordance with the ethical standards of the institutional and/or national research committee and with the 1964 Helsinki Declaration and its later amendments or comparable ethical standards. This study has been approved by the Medical Ethics Committee Brabant, the Netherlands (project number NL50258.028.14).

Provenance and peer review Not commissioned; externally peer reviewed.

Data sharing statement Data could be shared after consultation with the BIOS study group.

Open access This is an open access article distributed in accordance with the Creative Commons Attribution Non Commercial (CC BY-NC 4.0) license, which permits others to distribute, remix, adapt, build upon this work non-commercially, and license their derivative works on different terms, provided the original work is properly cited, appropriate credit is given, any changes made indicated, and the use is non-commercial. See: http://creativecommons.org/licenses/by-nc/4.0/.

\section{REFERENCES}

1. Johnell O, Kanis JA. An estimate of the worldwide prevalence, mortality and disability associated with hip fracture. Osteoporos Int 2004;15:897-902.

2. Hu F, Jiang $\mathrm{C}$, Shen J, et al. Preoperative predictors for mortality following hip fracture surgery: a systematic review and metaanalysis. Injury 2012;43:676-85.

3. Keene GS, Parker MJ, Pryor GA. Mortality and morbidity after hip fractures. BMJ 1993;307:1248-50.

4. Marks R. Hip fracture epidemiological trends, outcomes, and risk factors, 1970-2009. Int J Gen Med 2010;3:1-17.

5. Johnell $\mathrm{O}$. The socioeconomic burden of fractures: today and in the 21st century. Am J Med 1997;103:S20-S26.

6. Peeters CM, Visser E, Van de Ree CL, et al. Quality of life after hip fracture in the elderly: A systematic literature review. Injury 2016;47:1369-82.

7. De Vries J. Quality of life assessment. Assessment in behavioral medicine 2001:353-70.

8. Whoqol Group. Development of the World Health Organization WHOQOL-BREF quality of life assessment. The WHOQOL Group. Psychol Med 1998;28:551-8.

9. Grewal I, Lewis J, Flynn T, et al. Developing attributes for a generic quality of life measure for older people: preferences or capabilities? Soc Sci Med 2006;62:1891-901.

10. van Leeuwen KM, Bosmans JE, Jansen AP, et al. Comparing measurement properties of the EQ-5D-3L, ICECAP-O, and ASCOT in frail older adults. Value Health 2015;18:35-43.

11. Parsons N, Griffin XL, Achten J, et al. Outcome assessment after hip fracture: is EQ-5D the answer? Bone Joint Res 2014;3:69-75.

12. Xue QL. The frailty syndrome: definition and natural history. Clin Geriatr Med 2011;27:1-15.

13. Lin HS, Watts JN, Peel NM, et al. Frailty and post-operative outcomes in older surgical patients: a systematic review. BMC Geriatr 2016;16:157.

14. de Jongh MA, Kruithof N, Gosens T, et al. Prevalence, recovery patterns and predictors of quality of life and costs after non-fatal injury: the Brabant Injury Outcome Surveillance (BIOS) study. Inj Prev 2017;23(1):59.

15. von Elm E, Altman DG, Egger M, et al. The Strengthening the Reporting of Observational Studies in Epidemiology (STROBE) statement: guidelines for reporting observational studies. PLoS Med 2007; 4:e296.

16. Steverink N, Slaets J, Schuurmans H, et al. Measuring frailty: Development and testing of the groningen frailty indicator (GFI). Gerontologist 2001;41:236.

17. Peters LL, Boter H, Buskens E, et al. Measurement properties of the Groningen Frailty Indicator in home-dwelling and institutionalized elderly people. J Am Med Dir Assoc 2012;13:546-51.

18. EuroQol Group. EuroQol--a new facility for the measurement of health-related quality of life. Health Policy 1990;16:199-208.

19. Lamers LM, McDonnell J, Stalmeier PF, et al. The Dutch tariff: results and arguments for an effective design for national EQ-5D valuation studies. Health Econ 2006;15:1121-32.

20. Coast J, Flynn TN, Natarajan L, et al. Valuing the ICECAP capability index for older people. Soc Sci Med 2008;67:874-82.

21. Makai $P$, Koopmanschap MA, Brouwer WB, et al. A validation of the ICECAP-O in a population of post-hospitalized older people in the Netherlands. Health Qual Life Outcomes 2013;11:57.

22. Couzner L, Ratcliffe J, Lester L, et al. Measuring and valuing quality of life for public health research: application of the ICECAP-O capability index in the Australian general population. Int $J$ Public Health 2013;58:367-76.

23. Coast J, Peters TJ, Natarajan L, et al. An assessment of the construct validity of the descriptive system for the ICECAP capability measure for older people. Qual Life Res 2008;17:967-76.

24. Makai P, Brouwer WB, Koopmanschap MA, et al. Capabilities and quality of life in Dutch psycho-geriatric nursing homes: an exploratory study using a proxy version of the ICECAP-O. Qual Life Res 2012;21:801-12.

25. Van Buuren S. Flexible imputation of missing data. CRC press 2012.

26. Parsons N, Griffin XL, Achten J, et al. Modelling and estimation of health-related quality of life after hip fracture: A re-analysis of data from a prospective cohort study. Bone Joint Res 2018;7:1-5.

27. Twisk JW. Applied longitudinal data analysis for epidemiology: A practical guide. Cambridge University Press 2013.

28. Kistler EA, Nicholas JA, Kates SL, et al. Frailty and Short-Term Outcomes in Patients With Hip Fracture. Geriatr Orthop Surg Rehabil 2015;6:209-14

29. Patel KV, Brennan KL, Brennan ML, et al. Association of a Modified Frailty Index With Mortality After Femoral Neck Fracture in Patients Aged 60 Years and Older. Clin Ortho Rel Res 2014;472:1010-7.

30. Dayama A, Olorunfemi $O$, Greenbaum S, et al. Impact of frailty on outcomes in geriatric femoral neck fracture management: An analysis of national surgical quality improvement program dataset. Int J Surg 2016;28:185-90.

31. Griffin XL, Parsons N, Achten J, et al. Recovery of health-related quality of life in a United Kingdom hip fracture population. The Warwick Hip Trauma Evaluation--a prospective cohort study. Bone Joint J 2015;97-B:372-82.

32. Abimanyi-Ochom J, Watts JJ, Borgström F, et al. Changes in quality of life associated with fragility fractures: Australian arm of the International Cost and Utility Related to Osteoporotic Fractures Study (AusICUROS). Osteoporos Int 2015;26:1781-90.

33. Svedbom A, Borgström F, Hernlund E, et al. Quality of life after hip, vertebral, and distal forearm fragility fractures measured using the EQ-5D-3L, EQ-VAS, and time-trade-off: results from the ICUROS. Qual Life Res 2018;27:707-16.

34. Walters SJ, Brazier JE. Comparison of the minimally important difference for two health state utility measures: EQ-5D and SF-6D. Qual Life Res 2005;14:1523-32.

35. Cristancho P, Lenze EJ, Avidan MS, et al. Trajectories of depressive symptoms after hip fracture. Psychol Med 2016;46:1413-25.

36. Flikweert ER, Wendt KW, Diercks RL, et al. Complications after hip fracture surgery: are they preventable? Eur J Trauma Emerg Surg 2018;44:1-8.

37. Gabbe BJ, Lyons RA, Sutherland AM, et al. Level of agreement between patient and proxy responses to the EQ-5D health questionnaire 12 months after injury. J Trauma Acute Care Surg 2012;72:1102-5. 